\title{
perifèria
}

Número 6, Julio 2007

www. periferia. name

\section{Propuesta de materiales para atención en salud mental en un contexto intercultural}

\author{
Sanjuán, L L .- GRAFO (Grup de Recerca en Antropología Fonamental i \\ Orientada) - Universitat Autònoma de Barcelona ${ }^{2}$
}

\section{Resumen}

Se ha tratado de elaborar una serie de instrumentos adaptados a un modelo específico de atención en salud mental con personas en situación de diferencia cultural y/o riesgo de marginación social que defiende y trata de llevar a la práctica un tipo de asistencia que preste especial atención a todas aquellas cuestiones de índole cultural, social, política o económica que puedan relacionarse con la salud de las personas (especialmente la salud mental). El sistema sanitario se encuentra ante el reto de ofrecer una atención adaptada y eficaz a personas procedentes de entornos culturales diferentes al mayoritario, con representaciones de la salud y enfermedad en ocasiones alejadas a las que solían ser habituales en las consultas hasta no hace mucho. Estas representaciones condicionan de manera determinante el proceso de atención e influyen en el estado de salud de la población.

El modelo de atención que se presenta responde a la lectura crítica de bibliografía especializada, nacional e internacional, a la revisión exhaustiva de historias clínicas de los últimos años de profesionales del Servei de Psiquiatria del Hospital Vall d'Hebron y al trabajo de observación y participación desarrollado durante meses en el "Programa de Psiquiatría Transcultural-PsiTrans". Como resultado de este trabajo, se han elaborado una serie de documentos, aún preliminares y en fase de contrastación, que pretenden servir como instrumentos adaptados para la práctica asistencial en el Programa y en la información a los posibles usuarios.

Los documentos que se utilizarán en las consultas son los siguientes: Trípticos informativos, Consentimiento informado, Modelo de historia clínica de orientación sociocultural.

Palabras clave: Modelos de salud y enfermedad, modelos de atención de la salud, salud mental, minorías, marginación social, historia clínica de orientación sociocultural.

\footnotetext{
${ }^{1}$ Con la colaboración de los Drs. Francisco Collazos y Adil Qureshi (equipo de Psiquiatría Transcultural del Hospital Vall d'Hebron), la Dra. Teresa San Román (catedrática de Antropología de la UAB) y el GRAFO (Grup de Recerca en antropología Fonamental i Orientada, de la UAB). Parte de la elaboración de este documento ha sido posible gracias a una beca de investigación otorgada por la Fundació Jaume Bofill a Lucía Sanjuán durante el año 2005. La autora quiere agradecer a la Fundació su ayuda en este sentido.

${ }^{2}$ Enviar correspondencia a: Lucía Sanjuán, lucia_sanjuan@campus.uab.es
} 


\title{
perifèria
}

Número 6, Julio 2007

www. periferia. name

\begin{abstract}
The aim of this preliminary research has been to create several forms adapted to a specific model of medical attention regarding mental health for people culturally different and/or under risk of social marginalization. This model defends and tries to put into practice a sort of assistance in which special attention is paid to every cultural, social, political or economic variable that can be related to people's health (specially mental health). The current Health Care System is nowadays facing the challenge of offering effective attention to people with different cultural backgrounds. Moreover, the representations of health and disease have changed very fast and those representations determine in a very significant way both the Medical Care Process and the population's levels of health.
\end{abstract}

The model of attention here presented has been developed upon a critical reading of specialized bibliography, a thorough revision of clinical histories by the professionals working at Vall d'Hebron Hospital Psychiatric service (Servei de Psiquiatria de l'Hospital Vall d'Hebron), and the participant observation done during several months in the Program in Transcultural Psychiatry: Psi-trans (Programa de Psiquiatría Transcultural-PsiTrans). As a result, several documents have been elaborated, still in the pilot test phase, which try to be adapted to the medical practice.

Documents to be used in the offices are the following: Information brochure, Informed consent, Model of socio culturally oriented clinical history.

Keywords: models of health and disease, models of care attention, mental health, social exclusion, Model of socio culturally oriented clinical history.

\section{Trípticos informativos}

Los modelos de tríptico informativo que se presentan tienen como objetivo ofrecer información sobre la existencia, el tipo de atención y los criterios de accesibilidad del "Programa de Psiquiatría Transcultural-PsiTrans" del Hospital Vall d'Hebron. Con esta finalidad se han diseñado tres versiones diferentes: una para la Administración, otra para profesionales sanitarios y otra para pacientes.

\subsection{Modelo para la Administración ${ }^{3}$}

El documento para la Administración se entregará a profesionales encargados de la gestión de los servicios sanitarios, de bienestar social, expertos en asistencia

\footnotetext{
${ }^{3}$ Aunque aquí se presenta en el formato DIN-A4 habitual para facilitar la lectura, la impresión de todos los modelos se haría en formato tríptico.
} 


\title{
perifèria
}

\author{
Número 6, Julio 2007 \\ www.periferia.name
}

sanitaria a inmigrantes y decisores, de modo que dispongan de información sobre el tipo de atención que se pretende desarrollar y su utilidad. El texto del tríptico sería:

\author{
Nombre \\ Programa de Psiquiatría Transcultural-PsiTrans del Servei de Psiquiatria del Hospital \\ Universitari de la Vall d'Hebron.
}

\section{Presentación}

El "Programa de Psiquiatría Transcultural-PsiTrans" es un servicio del ICS dependiente del Servei de Psiquiatria del Hospital Vall d'Hebron que ofrece atención especializada en salud mental a población en situación de diferencia cultural y/o riesgo de marginación social. Además, el equipo pretende contar con la asesoría de personal especializado en antropología, antropología de la salud y etnografía de diversos grupos étnicos.

\section{Explicación del modelo de atención}

El "Programa de Psiquiatría Transcultural-PsiTrans" defiende la aplicación de un modelo de atención sanitaria integral en el que se tengan en cuenta todas aquellas variables -incluidas las de índole económica, social o cultural- que puedan tener una influencia en la salud mental de la población a la que se atiende.

Por ello, se desarrolla un modelo de asistencia en el que se presta especial atención al conocimiento del contexto etnográfico en el que se desarrolla el proceso terapéutico, algo necesario para la adecuación de los dispositivos sanitarios y la mejora de la planificación y ejecución de los programas orientados a la prevención y promoción de la salud de pacientes en situación de diferencia cultural y riesgo de marginación social.

La ubicación del Programa hace que sea posible el ingreso hospitalario en aquellos casos que lo requieran y facilita la realización de pruebas diagnósticas o la interconsulta con otras especialidades.

Desde el equipo del Programa no se contempla convertirse en un servicio paralelo de atención, ni la sustitución de otros servicios, sino ofrecer un servicio especializado, dentro de la red normalizada, que, por tener los recursos adecuados para ello, está en condiciones de dar respuesta a las necesidades específicas de un conjunto de personas que tienen dificultades para acceder y manejarse en los servicios de salud o para establecer una comunicación adecuada con los profesionales que le atienden.

\section{Descripción del modelo}

Con la intención de garantizar una atención adecuada a la salud independientemente de la pertenencia étnica y la adscripción cultural de los pacientes, se desarrolla un modelo de atención que abarca varios ámbitos:

- Ámbito médico-asistencial: Atención psicológica y psiquiátrica.

- Ámbito de investigación: El Programa desarrolla investigaciones interdisciplinares (médica y antropológica) sobre la atención en salud mental, la diversidad cultural y la marginación social.

- Asesoría y supervisión: El Programa pretende servir de puente entre el ámbito hospitalario y el de primaria, ofreciendo sesiones de asesoría o de supervisión de casos concretos a profesionales de otros servicios.

- Otras actividades: El equipo del Programa realiza actividades de formación y divulgación en temas de atención sanitaria a pacientes de diferentes culturas y sobre mediación en el ámbito sanitario. 


\section{perifèria}

Número 6, Julio 2007

www.periferia. name

\section{Derivación}

El "Programa de Psiquiatría Transcultural-PsiTrans" puede recibir pacientes procedentes de derivaciones de los servicios de urgencias, de médicos de familia, psiquiatras o psicólogos de los CAP o Centros de Especialidades, especial - pero no exclusivamente- los del área asistencial del Hospital Vall d'Hebron.

\section{Contacto}

"Programa de Psiquiatría Transcultural-PsiTrans"

Departamento de Psiquiatría

Edificio de la antigua Escuela de Enfermería, 5a planta

Hospital Universitario Vall d'Hebron

Passeig de la Vall d'Hebrón, 119-129

Tfno: 934894294

\section{Responsables}

Dr. Francisco Collazos, médico psiquiatra

Dra. Mar Ramos, médico psiquiatra

Dr. Adil Qureshi, psicólogo

\subsection{Modelo para profesionales sanitarios}

El modelo para profesionales sanitarios sirve para informar de la existencia del servicio entre los potenciales derivadores, para que tengan información del tipo de pacientes que pueden enviar, cómo tienen que hacerlo y cuál es el modelo de atención del Programa. El texto del tríptico sería:

\section{Nombre}

Programa de Psiquiatría Transcultural-PsiTrans del Servei de Psiquiatria del Hospital Universitari de la Vall d'Hebron.

\section{Presentación}

El "Programa de Psiquiatría Transcultural-PsiTrans" es un servicio del ICS dependiente del Servei de Psiquiatria del Hospital Vall d'Hebron que ofrece atención especializada en salud mental a población en situación de diferencia cultural y/o riesgo de marginación social.

\section{Explicación del modelo de atención}

El modelo de atención que desarrolla el Programa está orientado a pacientes que por razones de su procedencia étnica o adscripción cultural o por cuestiones sociales o económicas derivadas del proceso migratorio o de la vulnerabilidad social presentan dificultades para su atención en los servicios habituales. Además, el equipo pretende contar con la colaboración de personal especializado en antropología de la salud y etnografía de diversos grupos étnicos. 


\section{perifèria}

Número 6, Julio 2007

www.periferia.name

La ubicación del Programa hace que sea posible el ingreso hospitalario en aquellos casos que lo requieran y facilita la realización de pruebas diagnósticas o la interconsulta con otras especialidades.

Todo esto permite que el Programa disponga de una serie de recursos que lo hacen idóneo para ofrecer apoyo a los dispositivos que, por distintas razones, carezcan de estos medios.

\section{Actividades}

Se desarrolla un modelo de atención que abarca varios ámbitos:

- Ámbito médico-asistencial: Atención psicológica y psiquiátrica.

- Ámbito de investigación: El Programa desarrolla investigaciones interdisciplinares (médica y antropológica) sobre la atención en salud mental, la diversidad cultural y la marginación social.

- Asesoría y supervisión: El Programa pretende servir de puente entre el ámbito hospitalario y el de primaria, ofreciendo sesiones de asesoría o de supervisión de casos concretos solicitadas por profesionales de otros servicios.

- Otras actividades: El equipo del Programa realiza actividades de formación y divulgación en temas de atención sanitaria a pacientes de diferentes culturas social y sobre mediación sanitaria.

\section{Derivación}

El "Programa de Psiquiatría Transcultural-PsiTrans" puede recibir derivaciones de los servicios de urgencias, médicos de familia, psiquiatras o psicólogos de los CAP o Centros de Especialidades, especial - pero no exclusivamente- los del área asistencial del Hospital Vall d'Hebron.

\section{Contacto}

"Programa de Psiquiatría Transcultural-PsiTrans"

Departamento de Psiquiatría

Edificio de la antigua Escuela de Enfermería, 5a planta

Hospital Universitario Vall d'Hebron

Passeig de la Vall d'Hebrón, 119-129

Tfno: 934894294

\section{Responsables}

Dr. Francisco Collazos, médico psiquiatra

Dra. Mar Ramos, médico psiquiatra

Dr. Adil Qureshi, psicólogo

\subsection{Modelo para pacientes inmigrantes}

El tríptico para pacientes inmigrantes es un documento breve, de redacción sencilla, con el que se pretende explicar el funcionamiento del servicio y cómo acceder a él. 


\section{perifèria}

Número 6, Julio 2007

www. periferia. name

Esta explicación por escrito en ningún caso excluye, sino que complementa y sirve de refuerzo, a la acogida en el Programa: además de la explicación verbal, el paciente tendrá la posibilidad de quedarse con información escrita para volver a consultarla en cualquier momento o solicitar su opinión a otras personas. Al mismo tiempo, este tríptico pretende dar a conocer el Programa, en idioma y términos comprensibles, entre la población susceptible de atención, por lo que está previsto traducir el texto a diversas lenguas, como inglés, francés, árabe, chino, paquistaní, ruso o rumano.

El tríptico informativo para pacientes se les entregaría, preferentemente, en el momento en el que se hace la derivación, cuando piden la cita (si lo hacen en persona) o, si lo anterior no fuera posible, el día de la primera consulta. Es preferible entregarlo previamente a la visita para que el paciente tenga tiempo de leerlo con detenimiento antes de acudir y para evitar, también, que ese día reciba un volumen excesivo de información o el contexto sanitario le haga sentirse intimidado para aceptar el modelo de atención sin haber sopesado las ventajas e inconvenientes.

Además, se repartirían ejemplares en diversos puntos a los que pueden acudir los potenciales pacientes, de modo que puedan recibir información respecto al Programa tanto dentro como fuera del ámbito asistencial. Los lugares preferentes de elección serían:

- Mostradores de acceso y salas de espera de los CAPs y centros de atención especializada y profesionales de estos centros.

- Centros de atención socio-sanitaria y legal a inmigrantes y profesionales encargados.

- Asociaciones de inmigrantes.

\section{Nombre}

“Programa de Psiquiatría Transcultural-PsiTrans”

¿Qué es? 


\section{perifèria}

Número 6, Julio 2007

www. periferia. name

El "Programa de Psiquiatría Transcultural-PsiTrans" es un servicio, público y gratuito, que ofrece atención en salud mental a personas inmigrantes y de diferentes culturas.

El Programa está formado por profesionales de la psiquiatría, psicología, antropología, enfermería y mediadoras culturales de diferentes países.

El "Programa de Psiquiatría Transcultural-PsiTrans" tiene en cuenta cuestiones como:

- Historia de la inmigración del paciente.

- Relación de las dificultades legales, económicas, laborales, etc. con la salud.

- Adaptación a la nueva situación en el país.

- Adaptación al sistema sanitario.

- Efectos sobre la salud de la discriminación o los estereotipos.

- Respeto a las diferencias en la manera de entender el cuerpo, las enfermedades y los tratamientos, tratando de que haya comprensión entre el médico y el paciente.

- Respeto a las diferencias religiosas o morales.

Teniendo en cuenta esto, se presta una atención:

- Amplia y en profundidad.

- Con tiempo.

- Adaptada a la cultura.

\section{¿Cómo funciona?}

Las consultas se organizan del siguiente modo:

- Siempre se da información sobre el funcionamiento del Programa, de palabra y por escrito, y se solicita la autorización de los pacientes para iniciar el diálogo y los tratamientos.

- Siempre atenderán a los pacientes un psiquiatra o un psicólogo, dependiendo de las necesidades de cada caso.

- Además, en el equipo colaboran personas especializadas en antropología de la salud que tratarán cuestiones relacionadas con la cultura y la inmigración que pueden ser útiles para entender el estado de salud de los pacientes. Estas consultas son voluntarias, pero son una parte muy importante del modelo de atención.

- Es necesario un compromiso serio de colaboración porque:

o la posibilidad de ayudar es menor si no hay confianza entre el médico y el paciente.

- los temas relacionados con las condiciones de vida y la inmigración se tratan en profundidad, por lo que las consultas duran más tiempo, sobre todo la primera.

o puede haber un número mayor de consultas que en otros servicios. 


\section{perifèria}

Número 6, Julio 2007

www. periferia. name

- Gracias a la colaboración de los pacientes, los miembros del equipo realizan trabajos científicos para mejorar la calidad de la atención a personas de diferentes culturas. De estas investigaciones se beneficiarán también otros pacientes.

- Se garantiza absolutamente que el personal guardará el secreto profesional y que todos los datos serán confidenciales.

- Nunca se exigirán datos del paciente que no quiera revelar.

- Nunca se pedirá documentación sobre la situación legal en el país.

- El servicio es gratuito.

\section{¿Cómo se puede acceder?}

- Por indicación del médico de familia.

- Por indicación del médico de urgencias.

- Por indicación del psiquiatra o psicólogo.

- No es necesario tener "papeles" (permiso de residencia y trabajo o nacionalidad) para venir a este servicio.

\section{¿Cómo se puede contactar?}

"Programa de Psiquiatría Transcultural-PsiTrans"

Departamento de Psiquiatría

Edificio de la antigua Escuela de Enfermería, 5a planta

Hospital Universitaria Vall d'Hebron

Passeig de la Vall d'Hebrón, 119-129

Tfno: 934894294

\section{Plano y forma de llegar}

Plano de la zona y forma de llegar.

\section{Consentimiento informado}

\subsection{Justificación y objetivos}

La especificidad del modelo de atención, junto con la interdisciplinariedad del equipo y el uso de cierto tipo de datos con fines de investigación, además del evidente derecho del paciente a expresar o no su conformidad con la asistencia que se le propone, lleva a plantear la necesidad de elaborar un texto de consentimiento informado similar a los que se utilizan en otras especialidades. Este documento tiene como objetivo salvaguardar los derechos legales del paciente y explicitar el tipo de atención que se plantea, asegurando que se rige por estrictos criterios éticos y deontológicos. 


\section{perifèria}

Número 6, Julio 2007

www.periferia. name

A través del consentimiento informado, el paciente tiene la posibilidad de expresar, verbalmente y por escrito, que ha entendido el protocolo de atención, que da su permiso para las sesiones y para la utilización de los datos con fines de investigación y que asegura su colaboración en el protocolo de atención. Por este motivo, el documento -que firmará el paciente o su responsable legal-, se redacta en primera persona y se acompañará de una copia traducida a diversos idiomas (inglés, francés, árabe, chino, paquistaní, ruso o rumano).

El documento de consentimiento informado se entrega al paciente el primer día de consulta. Un miembro del equipo, que ya le habrá explicado como discurrirán las consultas con ayuda del tríptico informativo, leerá y explicará el texto, dejando tiempo para que el paciente lo lea de nuevo en privado y plantee las preguntas que considere pertinentes, y le pedirá, después, que lo firme. Dado que es posible que alguno de los pacientes no sepa leer ni escribir (en ningún idioma), siempre se le leerá el contenido detenidamente y aclarando las dudas que puedan surgir.

\subsection{Modelo de consentimiento informado}

El médico de familia, psiquiatra de zona u otro profesional socio-sanitario me ha recomendado que siga tratamiento en el "Programa de Psiquiatría TransculturalPsiTrans".

\section{Modelo de atención}

El "Programa de Psiquiatría Transcultural-PsiTrans" es un servicio del ICS dependiente del Servei de Psiquiatria Transcultural del Hospital de la Vall d'Hebron que ofrece atención especializada en salud mental a población de diferentes culturas.

El Programa cuenta con un equipo formado por profesionales de la psiquiatría, psicología, antropología de la salud, mediadoras culturales y personal de enfermería administrativo. Además, me han informado de que el equipo cuenta con personas especializadas en antropología de la salud con las que trataré aspectos relacionados con mi cultura y mi experiencia vital que pueden ser útiles para comprender mejor mi estado de salud. El equipo me ha informado de que estas consultas son voluntarias.

\section{I nvestigación}




\title{
perifèria
}

\author{
Número 6, Julio 2007 \\ www. periferia. name
}

\begin{abstract}
Los profesionales del "Programa de Psiquiatría Transcultural-PsiTrans" me han explicado que creen que es necesario mejorar los conocimientos existentes sobre la atención en salud mental a personas de diferentes culturas y que tienen, a veces, problemas sociales o económicos, por lo que uno de sus objetivos es realizar investigaciones científicas sobre este tema. Me han explicado también que la intención de estos trabajos es ayudar a que la asistencia médica sea mejor y que para esto, algunos de los datos obtenidos durante las consultas pueden ser de gran utilidad. Por este motivo, en ocasiones, solicitarán mi permiso para grabar o filmar las entrevistas, pero sólo las utilizarán para este propósito.
\end{abstract}

Además, me han informado de que es posible que sea útil (para mi tratamiento y para la investigación) hacer analíticas sanguíneas u otras pruebas, para lo que también solicitarán mi autorización. Me han explicado que tanto las grabaciones como las pruebas sólo se realizarán si doy mi consentimiento.

\section{Confidencialidad}

El equipo del "Programa de Psiquiatría Transcultural-PsiTrans" me ha asegurado que toda la información que proporcione a lo largo de las visitas, incluida la que se utilice para investigación, será tratada de manera confidencial. Esto quiere decir que tengo la garantía del equipo de que mis datos personales no serán utilizados por ellos fuera de las consultas y sólo para las investigaciones autorizadas. Además, los datos referidos al estado de mi regularización jurídica no se facilitarán en ningún caso a ninguna persona ajena a la Unidad, policía, gobierno o agencia.

\section{Compromiso}

Los profesionales del equipo me han informado de que éste es un servicio especializado poco común para el que se ha reunido a un equipo de profesionales de diferentes campos, dedicados específicamente a este trabajo y que esto supone unos costes económicos especiales para el sistema sanitario.

Por este motivo, el equipo del Programa me ha solicitado un compromiso serio de colaboración para que el uso de los recursos públicos sea el más adecuado y responsable.

\section{Autorización}

Declaro que el equipo del "Programa de Psiquiatría Transcultural-PsiTrans" me ha informado, de palabra y por escrito, del modo de funcionamiento de este servicio y he tenido ocasión de solucionar con ellos mis dudas al respecto.

Estoy de acuerdo en recibir atención en este Programa y colaboraré en el desarrollo de las consultas y entrevistas. He sido informado de que, en cualquier momento y sin que sea necesaria ninguna explicación, puedo retirar mi autorización. 


\section{perifèria}

Número 6, Julio 2007

www.periferia. name

\begin{tabular}{|c|c|c|}
\hline \multicolumn{3}{|c|}{ Apellidos y nombre del paciente: } \\
\hline NHC: & DNI o pasaporte: & Fecha: \\
\hline \multicolumn{3}{|c|}{ Apellidos y nombre de la persona responsable ${ }^{1}$ : } \\
\hline \multicolumn{3}{|l|}{ DNI: } \\
\hline \multicolumn{3}{|c|}{ Relación con el paciente: } \\
\hline \multicolumn{3}{|c|}{ Servicio: “Programa de Psiquiatría Transcultural” } \\
\hline \multicolumn{3}{|c|}{ Apellidos y nombre del médico que informa: } \\
\hline \multicolumn{3}{|l|}{ Número de colegiado: } \\
\hline Firma del paciente: & & co: \\
\hline
\end{tabular}

1. En caso de que el paciente sea incapaz de dar su consentimiento

\section{Modelo adaptado de historia clínica}

\subsection{Justificación y objetivos generales}

Se ha intentado elaborar un modelo de historia clínica que se incluya en el marco de un protocolo específico de atención en salud mental a pacientes en situación de diferencia cultural y riesgo de marginación social que responda a una manera de entender la atención psiquiátrica en la que se tienen presentes aquellas variables biográficas, económicas, socioculturales y políticas que pueden tener un papel en la salud de los individuos. 


\section{perifèria}

Número 6, Julio 2007

www. periferia. name

De acuerdo con este planteamiento, la propuesta inicial consiste en dividir el documento que conformará la historia de cada paciente en cuatro partes, que constituyen cuatro documentos relativamente independientes:

o Documento administrativo

o Historia clínica

o Historia sociocultural y migratoria

o Evaluación de la atención

Los objetivos generales que justifican este diseño son, en primer lugar, sistematizar la recogida de información, de forma que, sin perder de vista el objetivo fundamental de orientar el modelo de historia a la clínica, no se pierda la oportunidad de recoger datos sobre el contexto en el que se inscribe la atención, lo que facilitará simultáneamente el análisis de la información y la preservación de la significación que tiene para el paciente. En segundo lugar, y dada la composición interdisciplinar del equipo, con personal ajeno al ámbito sanitario, la división de la historia en documentos independientes permite mantener las garantías de acceso legal y ético a los datos, de manera que el apartado de información clínica sea tan sólo accesible al personal cualificado y autorizado a manejarla. Al mismo tiempo, la división no impide en absoluto que el documento mantenga una coherencia en su conjunto, ya que se entiende que los datos de índole sociocultural resultarán útiles tanto para el abordaje terapéutico propiamente dicho como para desarrollar investigaciones orientadas a mejorar la calidad de la atención a este tipo de usuarios.

En cuanto al tipo de datos, el diseño abierto de la mayoría de las preguntas garantiza la recogida de información cualitativa, mientras que su codificación posterior permitirá la obtención de datos cuantitativos.

Desde el punto de vista metodológico, es necesario puntualizar que el documento que aquí se presenta no constituye un guión de entrevista en el sentido en el que se emplea esta técnica en antropología, ni tampoco, exactamente, una historia clínica de las usadas en medicina, sino un documento que, respetando el modelo y 


\section{perifèria}

Número 6, Julio 2007

www. periferia. name

contenido básicos que deben tener las historias clínicas en el ámbito sanitario, tenga un enfoque de carácter sociocultural, recogiendo variables que no suelen estar presentes en las historias convencionales. Debe entenderse, además, como un "documento de máximos", de cumplimentación a medio o largo plazo y en sesiones orientadas prioritariamente al proceso terapéutico, conformando entonces un instrumento mediante el que se recogería, idealmente, una gran cantidad de información, aunque sabiendo que, en condiciones reales, pocas veces será posible recoger todos los datos para todos los pacientes. Lo que el modelo intenta es dejar abierta la posibilidad de recoger tanta información potencial y previsiblemente pertinente como sea viable, sin pretender convertirse en una guía de entrevista protocolizada o una encuesta cerrada, algo que iría en contra de su uso en el ámbito médico. Cabe aclarar, además, que el texto está diseñado para su uso conjunto por parte de personal sanitario y de antropología, de manera que la cumplimentación de la historia en su conjunto, de manera completa e ideal al menos, necesita personal especializado en cada uno de sus apartados. Obviamente, la sección de la historia que constituye la parte clínica está reservada, en exclusiva, a personal sanitario pero, de la misma manera, no puede esperarse que este tipo de profesionales esté en condiciones de explotar en toda su capacidad la sección de índole socio-cultural, para lo que es imprescindible personal especializado en antropología y antropología de la salud.

En cuanto al diseño, básicamente, se trata de crear una carpeta (en soporte papel e informático) correspondiente a los pacientes del Programa de Psiquiatría Transcultural-PsiTrans. En su interior se abrirá otra (con su nombre) para cada uno de los pacientes a los que se abra historia clínica, que contendrá, a su vez, los cuatro documentos en los que se recogerá la totalidad de la información. Esquemáticamente:

- Carpeta: Programa de Psiquiatría Transcultural-PsiTrans

o Carpeta "Nombre del paciente"

- Documento administrativo

- Historia clínica 


\section{perifèria}

Número 6, Julio 2007

www. periferia. name

- Historia migratoria y sociocultural

- Evaluación de la atención

Con el objetivo de garantizar su adecuación para la práctica clínica, se espera aplicar el modelo de historia en el Programa de Psiquiatría Transcultural-PsiTrans y, a medio plazo, si es posible, en otros centros, tanto de atención específica a inmigrantes como para población general, lo que permitiría un ajuste progresivo y una contrastación amplia del modelo.

Finalmente, debe quedar establecido que toda la recogida de datos estará supeditada a la estabilidad psicológica del paciente y su estado de salud, a su consentimiento explícito y al grado de confianza adquirida con los miembros del equipo.

\subsection{Modelo de historia clínica}

Se presenta a continuación el modelo de historia clínica, en todo caso abierto, incluso cuando una puesta a prueba, aún necesaria, mediante su aplicación práctica en la consulta, pueda darnos mayor confianza en su calidad y utilidad. Cabe aclarar también que esta versión sufrirá ciertas modificaciones en cuanto a su diseño visual, que se han omitido en este momento para facilitar su lectura: no están activados los recuadros correspondientes a los formularios y la información respecto al contenido de las preguntas, que aquí se presenta escrita tras su enunciado, se encontrará en modo de cuadro de comentario sólo visible al situarse sobre el número de la pregunta. Además, se diseñará un mapa del documento, situado a la izquierda de la pantalla, que permitirá un desplazamiento rápido por el interior de la historia.

\section{HI STORI A I : DOCUMENTO ADMI NI STRATI VO}

El objetivo de este documento es facilitar la recogida y el acceso posterior a los datos sociodemográficos del paciente. 


\section{perifèria}

Número 6, Julio 2007

www.periferia. name

La información correspondiente a este apartado puede ser recogida por personal de enfermería o administrativo adecuadamente entrenado, por lo que se contempla la posibilidad de desarrollar sesiones de formación para estos profesionales destinadas a la adquisición de habilidades de comunicación con los usuarios del Programa.

Incluye dos grupos de datos:

A. Datos sociodemográficos del paciente

B. Datos de la consulta

\section{A. DATOS SOCI ODEMOGRÁFI COS BÁSI COS}

1. Nombre y apellidos

Apellidos, Nombres

\section{Número de historia de la Unidad}

Número según formato de la Unidad: 001, 002, 003...

\section{Número de historia clínica}

Número según formato habitual en HUVH

\section{Género}

Hombre

Mujer

\section{Fecha de nacimiento}

$\mathrm{dd} / \mathrm{mm} /$ aaaa

En caso de que no sea posible recoger la fecha exacta, tomar la edad aproximada y codificarla según formato.

Calcular la edad en el momento de inicio de la consulta.

\section{Lugar de nacimiento}

Recoger:

- País

- Ciudad

- Especificar región o zona de procedencia (norte, sur, urbano, rural...)

\section{Lengua en que prefiere que se realice la consulta}

Recoger la lengua que el paciente prefiere utilizar en la consulta, para incrementar las posibilidades de comunicación. 


\section{perifèria}

Número 6, Julio 2007

www.periferia. name

\section{Dirección y teléfono de contacto}

Recoger, en la medida de lo posible, un modo de localizar al paciente en caso de que sea necesario. Recoger el nombre del barrio y, si se conoce, el distrito de Barcelona al que pertenece. Si es una ciudad fuera de Barcelona, especificar la ciudad y el barrio.

\section{B. DATOS DE LA CONSULTA}

\section{Procedencia de la derivación}

Especificar el centro y profesional que realiza la derivación al Programa: médico de familia, psicólogo o psiquiatra de zona, servicio de urgencias, etc.

Si es posible, recoger el texto en el que se solicita la derivación.

\section{Fecha de la primera visita}

$\mathrm{dd} / \mathrm{mm} /$ aaaa

\section{Calendario de consultas}

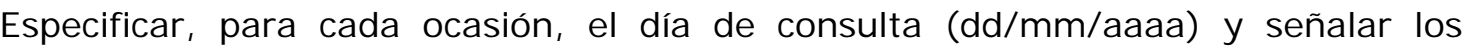
miembros del equipo que ven al paciente. Si anula o no acude a la consulta, señalarlo en la fecha correspondiente. Dejar indicado la fecha correspondiente a la próxima consulta programada.

\section{HISTORI A I I : HISTORI A CLÍ NI CA}

Se trata de un documento similar a cualquier historia clínica convencional en psiquiatría y psicología. Incluye cinco apartados diferenciados, de los que se responsabiliza, exclusivamente, el personal sanitario:

\section{HI STORI A CLÍ NI CA NO PSI QUI ÁTRI CA}

Recoge los datos habituales de cualquier modelo de historia clínica: antecedentes personales y familiares, exploración física, etc.

\section{Antecedentes somáticos personales. Alergias, cirugía, hospitalizaciones}

Recoger información sobre antecedentes somáticos de interés.

\section{Exploración física básica}

Recoger datos sobre talla, peso, tensión arterial y variaciones significativas a lo largo de la consulta.

\section{Patología somática actual}

Recoger datos clínicos sobre patología somática actual que sean de interés, incluyendo, si procede, la medicación que toma. 


\section{perifèria}

Número 6, Julio 2007

www. periferia. name

\section{HISTORI A PSI QUI ÁTRICA}

\section{Motivo de consulta}

Recoger la narración del paciente sobre la causa que motiva la consulta.

\section{Antecedentes psiquiátricos personales}

Recoger información sobre antecedentes psiquiátricos personales, especificando, si procede, si se han producido antes o después de la emigración y tipo de intervención que se realizó.

\section{Antecedentes psiquiátricos familiares}

Recoger información sobre antecedentes psiquiátricos familiares, teniendo en cuenta la concepción particular de familia y enfermedad psiquiátrica que pueda tener el paciente.

\section{Tratamiento psiquiátrico actual}

Se refiere a tratamiento previo a la consulta en el Programa.

\section{Hábitos tóxicos. Sustancias legales}

Recoger información sobre consumo de alcohol, tabaco y fármacos, momento de inicio, patrón actual de consumo, consecuencias atribuidas al mismo y solicitud de tratamiento.

\section{Hábitos tóxicos. Sustancias ilegales}

Recoger información sobre consumo de sustancias ilegales (cannabis, cocaína, heroína u otras sustancias), momento de inicio, patrón actual de consumo, consecuencias atribuidas al mismo y solicitud de tratamiento.

\section{Hábitos tóxicos. Otro tipo de sustancias}

Hace referencia a preparados usados en el lugar de origen pero no conocidos (o usados) aquí. Recoger nombre del producto, vía de consumo, efectos deseados e indeseables, modo de obtenerlo, etc.

\section{Hábitos alimentarios}

Recoger, de manera genérica, aspectos relacionados con la alimentación que puedan ser de relevancia para la consulta.

\section{Sexualidad}

Recoger, de manera genérica, aspectos relacionados con la sexualidad del paciente que puedan ser de relevancia para la consulta o de preocupación o extrañeza para el paciente.

\section{Rasgos de personalidad. Autodefinición}

Recoger relato del paciente sobre modo en el que se ve a sí mismo.

\section{Exploración psicopatológica}




\section{perifèria}

Número 6, Julio 2007

www.periferia. name

Se recogen los datos habituales en este tipo de exploración: actitud, estado de la consciencia, conducta motora, percepción, memoria, orientación, atención, sueño, afectividad, lenguaje, curso del pensamiento, contenido del pensamiento y otros datos de interés.

\section{Exploraciones complementarias}

Recoger información sobre la realización, en el Programa, de pruebas complementarias: analítica sanguínea, estudio genético, etc.

\section{Enfermedad actual}

Se recoge en este apartado la impresión diagnóstica del profesional.

\section{Diagnóstico}

Se sigue el sistema de clasificación multiaxial del DSMIV. Si se valora conveniente, puede añadirse el diagnóstico de acuerdo al ICE-10 o cualquier comentario relativo a la adscripción diagnóstica.

EJE I: Trastornos clínicos. Otros problemas que pueden ser objeto de atención

EJ E II: Trastornos de la personalidad. Retraso mental

EJ E III : Enfermedades médicas

EJ E IV: Problemas psicosociales y ambientales

EJ E V: Escala de evaluación de la actividad global

\section{Tratamiento}

Tratamiento prescrito por el profesional.

30.Evolución 


\section{perifèria}

Número 6, Julio 2007

www.periferia.name

Narración por parte del profesional de la evolución clínica del paciente. Registrar la fecha en la que se realiza cada anotación.

\section{E. HISTORI A PSI COLÓGI CA}

Se diferencia la historia psicológica de la psiquiátrica en la medida en que ciertos pacientes tendrán consultas independientes con el psiquiatra y psicólogo y otros sólo con uno de ellos. El modelo que se ofrece de historia psicológica pretende ser un vehículo de seguimiento de la terapia indicada al paciente y está adaptado a las preferencias individuales del psicólogo del equipo.

\section{Escalas de valoración psicológica}

Se intentarán pasar a los pacientes diversos tipos de escalas de valoración psicológica: MEIM2, BIS, PAS, PHQ, SEMI, WAI, IPQ-R, etc. Obviamente, no se pasarán todas a todos los pacientes, sino tan sólo aquellas que se valoren como relevantes para los objetivos de la atención sanitaria y la investigación y que sean aceptadas por el paciente.

Se registra el tipo concreto de escala que se pase a cada paciente y un informe de los resultados de cada una de ellas.

\section{Valoración subjetiva del paciente de su propio estado}

Recoge la narración personal del paciente.

\section{Valoración psicológica}

Recoge datos, del profesional, sobre valoración psicológica del paciente.

\section{Estado de ánimo. Afectividad}

Recoge datos la valoración del profesional.

\section{Planificación}

Se refiere a la planificación de la terapia por parte del profesional. Se fechará cada anotación.

\section{F. FORMULACIÓN CULTURAL}

Sigue el modelo establecido por el DSM-IV, aunque sólo se recogen en este momento algunas preguntas respecto a la relación del diagnóstico con la cultura del paciente y su historia migratoria. El resto de aspectos considerados en el DSMIV se tratan en el documento de historia sociocultural y su inclusión en estos apartados responde a criterios de coherencia interna de los documentos, continuidad argumental de las preguntas y garantías de profundidad en su abordaje.

36.Especificidad del diagnóstico en relación con la cultura del paciente 


\section{perifèria}

Número 6, Julio 2007

www. periferia. name

Recoge la valoración subjetiva (pero concreta y fundamentada) por parte del equipo. Se trata de una valoración por parte de personal sanitario, no de antropología, de manera que es necesario tener en cuenta el posible error al que pueda conducir la disimetría entre las concepciones culturales del paciente y su médico. El contenido de esta pregunta debe, en cualquier caso, contrastarse adecuadamente con la información obtenida a partir de la historia sociocultural y migratoria.

\section{Relación del diagnóstico con el proceso migratorio}

Recoge la valoración subjetiva (pero concreta y fundamentada) por parte del equipo. Deben recogerse los elementos concretos del proceso migratorio que se relacionen de forma clara con el diagnóstico.

\section{Valoración del grado de cumplimiento del tratamiento y motivos que lo justifican}

Recoge la valoración por parte del equipo respecto al grado de cumplimiento de las indicaciones terapéuticas por parte del paciente. Recoger información especialmente sobre posible relación del incumplimiento con factores socioculturales, económicos y de accesibilidad del sistema sanitario.

\section{HI STORI A I I I : HI STORI A SOCI OCULTURAL Y MI GRATORI A}

En este documento el objetivo consiste en tratar de protocolizar la recogida de todos aquellos aspectos no exclusivamente clínicos y de especial interés sociocultural que sean útiles para un mejor abordaje terapéutico del paciente y para la investigación clínica y antropológica en este ámbito. Su cumplimentación recae fundamentalmente en personal especializado en antropología de la salud, aunque será accesible a personal sanitario.

Este documento recoge, a veces de manera literal, cuestiones ya planteadas en el Guión Sociocultural General, para la orientación del trabajo de campo, de T. San Román y en el Guión sobre la Salud y Atención Sanitaria, presentado antes.

\section{H. FAMI LI A}

\section{Estado civil}

Recoger información sobre estado civil: soltero, casado, separado, divorciado, viudo y otras posibles categorías.

Si es posible, recoger cuestiones relacionadas con el tipo de matrimonio (civil, religioso, monógamo, polígamo...), legalidad de la unión, lugar en el que se realizó, tipo de rito, etc.

Si es posible, especificar la procedencia de la(s) pareja(s) y su lugar de residencia actual. 


\section{perifèria}

Número 6, Julio 2007

www. periferia. name

Si procede, especificar criterios de elección de la pareja

En caso de estar separado, especificar las circunstancias e implicaciones de la separación.

\section{Número de hijos vivos}

Recoger:

- Número, género y edad.

- Lugar de residencia, especificar, si procede, ciudad concreta y motivos para residir en ella.

En caso de poligamia o diversos matrimonios, recoger información por separado para cada uno de ellos.

\section{I mportancia de las relaciones familiares en su vida}

Recoger cuestiones sobre:

- Papel que cree que desempeña en su familia.

- Significado de ser hijo, padre, marido, mujer...

- Esferas de la vida en las que considera importante el papel de la familia

- Decisiones que consulta a la familia o que se toman en conjunto (miembros concretos de la familia a los que consulta y motivos)

- Papel económico de la familia.

- Preguntar sobre grado de control respecto a la situación de su familia (unidad, situación económica, reparto de roles...).

\section{REDES SOCI ALES}

\section{Tipo y número de parientes emigrados del país de origen}

Se refiere al número y tipo de parientes emigrados del país de origen que pueden formar parte de la red social inmediata del paciente. Recoger información sobre tipo de parentesco, edad, género y tipo de relación. Recoger también, para cada uno de ellos, si residen en: misma vivienda, barrio, ciudad, en Catalunya o Estado español. Si procede, incluir datos sobre familiares emigrados en otros países.

\section{Redes de apoyo}

Recoger información sobre personas a las que acude, procedencia, tipo de relación, situaciones concretas y tipo concreta de ayuda, en función de: 


\section{perifèria}

Número 6, Julio 2007

www. periferia. name

- Redes de apoyo económico: tandas, préstamos, reciprocidad diferida o generalizada...

- Redes de apoyo para situaciones del ámbito legal, laboral, de vivienda...

- Redes de apoyo para ayuda doméstica, cuidado de hijos y ancianos de la casa...

- Otro tipo de ayuda: informaciones diversas...

No es necesario incluir redes de apoyo para la salud, se pregunta en documento correspondiente

\section{J. VI VI ENDA}

\section{Tipo de vivienda y tenencia}

Recoger tipo de vivienda (casa, piso, local...) y régimen de tenencia (propiedad, alquiler, cesión...). En caso de vivir en alquiler, especificar si es piso compartido o realquilado (habitación). Si es posible, recoger precio aproximado de la vivienda y porcentaje que le corresponde pagar.

45.Número de personas con quien convive. Tipo de relación (familiar, laboral, amistad, procedencia...)

Recoger número de personas que viven (de manera estable) en la misma vivienda y tipo de relación que les une.

46. Nivel de satisfacción con la vivienda actual. ¿Qué necesitaría para estar mejor?

Recoger información sobre:

- Disponibilidad y estado de los servicios básicos: agua, instalación de luz, gas o butano, baño y cocina

- Disponibilidad de espacio propio

- Valoración subjetiva del estado de la vivienda (humedad, luz natural, ruidos, etc.)

- Valoración subjetiva de la equipamiento de la vivienda (nevera, cocina, microondas, lavadora...)

- Comparación subjetiva con vivienda en país de origen

- Intención de cambio de vivienda actual y motivos

- Expectativas y deseos en cuanto a la vivienda y tipo de vivienda que desearía 


\section{perifèria}

Número 6, Julio 2007

www.periferia. name

- Valoración subjetiva de la relación entre las condiciones de su vivienda y su estado de salud

- Grado de control respecto a la elección de vivienda (localización, dimensiones, características de salubridad y comodidad, tipo de tenencia, estabilidad de la vivienda...).

\section{K. HISTORI A MI GRATORI A}

\section{Grupo étnico en el que se adscribe}

Recoger información sobre criterios que definen la pertenencia étnica e implicaciones de la misma.

\section{Regularización jurídica}

Recoger información sobre tipo de documentación disponible: ninguna, sólo empadronamiento, permiso de residencia y trabajo, nacionalidad...

Si es posible, recoger itinerario de obtención de los papeles y grado de control personal sobre el proceso de regularización.

\section{I tinerario migratorio}

Recoger información sobre:

- Motivo para la emigración

- Planificación previa a la emigración

Recoger, de manera aproximada, para cada uno de los países y ciudades españolas por las que pasó antes de llegar a Barcelona, fechas y motivos para la entrada y la salida.

- Criterios de elección de Barcelona como lugar de destino

- Posibilidad de continuar el itinerario hacia otro país o ciudad, motivos para hacerlo y planificación al respecto

- Experiencias de la emigración (personas que lo acompañaban, que le esperaban o con las que contactó, dificultades, etc.)

- Grado de control respecto al proceso migratorio (influencias importantes en la decisión de emigrar, de elección del lugar o del itinerario, de volver, etc.)

- Coste, endeudamiento en origen o en destino, plazos y forma de pago...

Si es posible, recoger información sobre: 


\section{perifèria}

Número 6, Julio 2007

www.periferia. name

- Número de veces que ha vuelto a su país de origen desde su salida y desde su llegada a Barcelona. Especificar motivos y tiempo de estancia

- Planificación concreta de retorno definitivo (acciones concretas, motivos y tiempo estimado) y expectativas ideales en este sentido

- Intención de reagrupamiento familiar en ciudad de acogida: motivo y familiares a los que traería

- Grado de control respecto a la estabilidad de la migración (factores de los que depende la decisión de volver, quedarse o traer a la familia, personas y circunstancias que influyen y control personal de la decisión)

50.Valoración subjetiva de pérdidas y ganancias a raíz de la emigración.

Recoger información sobre:

- Reconocimiento social y familiar de la emigración. Importancia de su emigración para su familia

- Aspectos positivos de la emigración y comparación con las expectativas previas

- Aspectos negativos de la emigración y comparación con las expectativas previas

- Estimación de si, en circunstancias similares, habría vuelto a emigrar y por qué

51.Valoración subjetiva de la relación entre su historia migratoria y su estado de salud

Especificar efectos o síntomas concretos a corto, medio y largo plazo, tanto físicos como psicológicos.

52.Discriminación o racismo percibidos. Circunstancias e influencia en su vida cotidiana y en su estado de salud

Recoger información sobre:

- Estereotipos que cree que la sociedad de acogida mantiene respecto a la de origen e influencia en su vida cotidiana

- Situaciones concretas de discriminación o racismo (diferenciando entre ambos) que ha vivido, influencia en su vida cotidiana y en su estado de salud

- Estereotipos del paciente respecto a la sociedad de acogida

L. TRABAJ O Y SITUACIÓN ECONÓMI CA

53.Situación laboral 


\section{perifèria}

Número 6, Julio 2007

www.periferia. name

Recoger las características de los trabajos desempeñados: descripción del trabajo, tipo de contrato, etc., diferenciando entre trabajo principal (en cuanto al nivel de ganancias y estabilidad) y secundarios.

\section{Nivel de satisfacción con la situación laboral actual. ¿Qué necesitaría para estar mejor?}

Recoger información sobre aspectos positivos y negativos en el trabajo, intención de cambio, motivos y posibilidades reales, etc. Recoger también grado de control individual respecto a la situación laboral y posibilidades de trabajo (factores que influyen en la elección y mantenimiento de un tipo de trabajo, personas y circunstancias que influyen y grado de control personal sobre la situación)

\section{Situación económica}

Recoger información sobre percepción de ayudas sociales, otros miembros de la unidad familiar que trabajen y aporten dinero al conjunto. Investigar también otras posibles fuentes de ingresos: alquileres, compensación de favores, pagos en especies, etc.

Recoger información sobre patrón de gasto: porcentaje de los ingresos dedicados a alquiler, deuda, remesas...

Recoger valoración subjetiva sobre si las ganancias totales son suficientes para cubrir los gastos que tienen que afrontar.

\section{Valoración subjetiva de la relación entre sus condiciones de trabajo y su estado de salud}

Atribuciones en función de: dureza física del trabajo, horario, ganancias, ambiente laboral, estabilidad laboral. Recoger síntomas concretos, tanto físicos como psicológicos, a corto, medio y largo plazo.

\section{FORMACIÓN}

\section{Escolarización}

Especificar estudios realizados en país de origen y fuera de él. Recoger información sobre: tipo de estudios, tipo de centro, años completos de escolarización. Especificar si la escolarización se interrumpía por temporadas (trabajo, cosecha, cuidado de hermanos...). Especificar nivel y/o competencias alcanzados.

Recoger estudios no reglados, actividades de formación profesional...

Valoración sobre papel de la formación en su vida y en sus condiciones de vida actuales: Recoger información sobre para qué han servido los estudios (trabajo, relaciones sociales, conocimiento de la sociedad de acogida...).

58.Nivel de conocimientos y ámbito de uso de lenguas 


\section{perifèria}

Número 6, Julio 2007

www. periferia. name

Especificar ámbitos en los que utiliza la lengua (laboral, familiar, vecinal, instituciones, etc.) y capacidad de manejo (entender, hablar palabras sueltas, conversaciones sencillas, leer, escribir...), para:

- Castellano

- Catalán

- Otras lenguas que conoce

\section{N. RELIGIÓN}

\section{Religión a la que se adscribe}

Especificar, si es posible, religión a la que se adscribe y grado de práctica. Pedir que especifique cambios que ha experimentado a raíz de la emigración y posibilidades reales para la práctica religiosa en la ciudad de acogida

\section{I nfluencia de la práctica religiosa en la salud}

Recoger información sobre influencia de la práctica adecuada de la religión en el mantenimiento o recuperación de la salud y de las transgresiones en el proceso de enfermar.

\section{Ñ. ACCESI BI LI DAD SI STEMA SANITARIO}

\section{Disponibilidad de tarjeta sanitaria}

Especificar si se dispone de tarjeta sanitaria y momento en que se obtuvo. Recoger información sobre itinerario de gestión: requisitos exigidos, dificultades, estrategias para obtenerla...

\section{Valoración subjetiva de la accesibilidad del Programa}

Recoger, de manera aproximada datos y valoración subjetiva de:

- Tiempo transcurrido desde que pide cita al derivador hasta que acude a la consulta

- Tiempo desde que solicita cita en el Programa hasta que es atendido

- Tiempo medio de espera desde que llega a las consultas del Programa hasta que es atendido

- Tiempo invertido en llegar desde su domicilio o lugar de trabajo a la consulta

- Medios de transporte utilizados

- Valoración subjetiva de la accesibilidad física: valoración del tiempo, complejidad para llegar, transbordos...

63.Valoración subjetiva de la accesibilidad del sistema sanitario 


\section{perifèria}

Número 6, Julio 2007

www.periferia. name

Recoger información sobre:

- Grado de conocimiento de los servicios: tipos de servicios, utilidad de cada uno de ellos, modos de acceso, etc. Recoger también el modo por el que conoció el Programa

- Valoración subjetiva de la capacidad de manejo en los diferentes tipos de servicios

- Necesidades percibidas para mejorar su capacidad de acceso. Si es posible, recoger el relato de situaciones concretas que justifiquen la valoración

- Percepción subjetiva de calidad del sistema sanitario. Especificar criterios en los que se basa la valoración

- Percepción de diferencias en la estructura, modo de funcionamiento, relación médico-paciente, etc. entre biomedicina en ciudad de acogida y país de origen. Comparación y balance de ventajas y defectos

- Valorar conocimiento respecto a los derechos en salud y percepción subjetiva de posibilidades de ejercerlos.

\section{O. REPRESENTACIÓN PERSONAL DE LA ENFERMEDAD (ACTUAL)}

64.¿Qué le pasa?, ¿qué nombre le da al problema que padece?

Se trata de recoger aquí sobre todo el nombre que se le da a la enfermedad. Recoger también el estado de salud autopercibido (físico y psíquico).

\section{5.¿Cuál es la causa?, ¿por qué cree que tiene este problema?}

Recoger información sobre:

- Atribución causal, relación de la enfermedad con su comportamiento o el de otros, sus condiciones de vida, biografía, etc.

- Fecha de comienzo de la enfermedad y motivo por el que cree que empezó en ese momento.

66.¿Cuáles son los síntomas?, ¿en qué nota que está enfermo?

Recoger datos sobre:

- Descripción personal (no medicalizada) de los síntomas

- Partes del cuerpo a las que ha afectado el problema de salud y temor a las que pueda afectar.

67.¿Cuál es el tratamiento adecuado?, ¿qué cree que hay que hacer (o qué hace) para que se sienta mejor? Compatibilidad con el tratamiento prescrito 


\section{perifèria}

Número 6, Julio 2007

www. periferia. name

Recoger información sobre:

- Tratamiento biomédico que considera adecuado

- Cambios en las condiciones o estilo de vida que cree que necesitaría para estar mejor.

- Compatibilidad del tratamiento prescrito en el Programa con sus posibilidades o condiciones de vida con la y representación personal de la enfermedad

- Posibilidad de que algún miembro de la familia o del entorno no esté de acuerdo con su participación en el Programa e influencia o autoridad sobre el seguimiento del tratamiento o las consultas.

\section{Repercusiones de su enfermedad}

Recoger datos sobre:

- Percepción de si el problema que padece es grave o no y por qué y según qué criterios

- Grado de control sobre su propio estado de salud

- Aspectos del problema actual que más preocupan al paciente

- Modo en que el problema afecta a su vida cotidiana. Especificar por ámbitos: trabajo, planes de futuro... Recoger el grado de prioridad de la salud respecto a otras necesidades (vivienda, papeles, trabajo...)

- Modo en que el problema afecta a las personas de su entorno. Especificar: familia aquí y en origen, miembros de la red social...

- Modo en el que problema influye en la manera en cómo los demás le ven y cómo se ve a sí mismo.

\section{Redes de apoyo en salud}

Recoger datos sobre:

- Personas a las que ha contado el problema que padece. Tipo de relación y motivos

- Tipo de ayuda que ha solicitado y que ha recibido y de quién (tipo de relación)

- Personas a las que ha decidido ocultar el problema que padece. Tipo de relación y motivos

- ¿Cree que la ayuda o el apoyo de los que le rodean serían diferentes si su enfermedad fuera de otro tipo? 


\section{perifèria}

Número 6, Julio 2007

www. periferia. name

- Red potencial de apoyo si hubiera ocurrido en el lugar de origen

P. I TI NERARI O EN RELACI ÓN AL PROBLEMA ACTUAL

70.I tinerario terapéutico para el problema actual

Recoger posibilidades de itinerario terapéutico:

- Remedios caseros

- Biomedicina (diferente o anterior al Programa)

- Medicina tradicional

- Medicinas alternativas (homeopatía, acupuntura...)

- Religión

- Otras terapias (cartas, limpieza del aura...)

Para cada los supuestos de itinerario terapéutico, recoger:

- Momento cronológico. Especificar si la consulta se produjo antes o después de la emigración (o ambos). En caso de consulta antes de la emigración, recoger información sobre influencia de la enfermedad y (necesidad de) tratamiento en la decisión de emigrar. Especificar si la consulta se produjo en ciudad de acogida o país de origen (o ambos).

- Tiempo transcurrido entre la aparición del problema y la decisión de buscar ayuda y motivos que lo justifican

- Criterios de elección del modelo de atención, del centro y del terapeuta concretos

- Modo en cómo ejerce cada terapeuta concreto

- Pago o contraprestación

- Nombre de la enfermedad y explicación causal que recibió.

- Tipo de tratamiento prescrito

- Grado y posibilidades de cumplimiento del tratamiento, especificando adecuación a las condiciones de vida, representación personal de la enfermedad, etc.

- Grado de satisfacción con el trato personal recibido. Pedir, específicamente, que señale los aspectos positivos y los negativos

- Grado de satisfacción con la eficacia de la intervención. 


\section{perifèria}

Número 6, Julio 2007

www. periferia.name

- Grado de control durante la intervención

- En caso de simultanear consultas, recoger el grado de compatibilidad (y especificidad) de las explicaciones y los tratamientos

- Valoración de la relación entre el tratamiento seguido y el cambio en el estado de salud, si procede

\section{HISTORI A I V: VALORACIÓN DE LA ATENCIÓN}

En este caso se trata de obtener información respecto a la valoración que el paciente y sus acompañantes hacen sobre el trato recibido en el Programa. Se pretende recoger no sólo sus opiniones respecto al modelo de atención y el tipo de relación establecida con los distintos miembros del equipo sino, fundamentalmente, la percepción subjetiva de la evolución (positiva o no) de su estado de salud y las atribuciones causales que realiza sobre ella en cada momento del tratamiento.

Esta información será especialmente útil para comprender mejor las representaciones en torno a la salud y la asistencia sanitaria de los pacientes y para valorar la capacidad del Programa para adaptar su estructura y funcionamiento a sus necesidades y características, permitiendo, si fuese necesario, reorientar el protocolo de atención. Así mismo, servirá de refuerzo del consentimiento del paciente al protocolo de atención.

El documento está formado por cuatro apartados que se cumplimentarán en diferentes etapas y a lo largo del tiempo, aunque se entiende que el proceso de evaluación será continuo a lo largo de todo el tratamiento (en la medida que las opiniones, actitudes e interpretaciones del paciente, además de su estado de salud, puedan ir modificándose):

\section{Q. A PRIORI : EXPECTATIVAS PREVI AS}

En la primera o segunda entrevista, se recogerán para evaluar las expectativas previas del paciente de cara a la asistencia en el Programa.

71. En esta Unidad, ¿qué cree que podemos hacer para ayudarle?

Recoge la narración subjetiva del paciente respecto a sus expectativas.

\section{R. DURANTE LA I NTERVENCIÓN}




\section{perifèria}

Número 6, Julio 2007

www.periferia. name

Hacia la mitad de las consultas (calculado a partir de las previsiones iniciales del equipo), cuando la confianza con el paciente lo permita, se recogerán datos sobre la evolución de la intervención: en qué medida se cumplen las expectativas previas y cuáles son las valoraciones respecto al protocolo de atención. El establecimiento de una fecha más o menos concreta pretende, tan sólo, garantizar que esta exploración se haga al menos una vez por cada paciente en el curso de su tratamiento (a parte de la inicial y final).

\section{Valoración del modelo de atención}

Recoger información sobre:

- Valoración del trato personal recibido (intromisión en la intimidad, relación con el equipo, etc.)

- Protocolo de tratamiento

- Grado de cumplimiento de expectativas previas

- Grado de control durante la intervención

- Pedir, específicamente, que señale los aspectos positivos y los negativos

73. Valoración del cambio en el estado de salud (mejoría o no) ¿A qué cree que se debe?

Tratar de recoger información sobre percepción de utilidad de las consultas y modo en cómo lo hacen

\section{S. AL ALTA: VALORACIÓN DEL PROTOCOLO DE ATENCIÓN}

En el momento del alta médica (o hacia el final del tratamiento) se recogerán, de manera más exhaustiva, las valoraciones del paciente respecto al trato y a la atención recibida, especialmente en relación con aquellos aspectos novedosos que ofrece el Programa, intentando también obtener datos de aquellos pacientes que acuden de manera irregular. También se recogerá la opinión de aquellas personas que hayan acompañado al paciente de manera regular a la consulta, sobre todo en relación a la atribución de causas a la mejoría (esperable) del paciente.

\section{Valoración del modelo de atención}

Recoger información sobre:

- Valoración del trato personal recibido (intromisión en la intimidad, relación con el equipo, etc.)

- Protocolo de tratamiento

- Grado de cumplimiento de expectativas previas 


\section{perifèria}

Número 6, Julio 2007

www.periferia. name

- Grado de control durante la intervención

- Pedir, específicamente, que señale los aspectos positivos y los negativos

75. Valoración del cambio en el estado de salud (mejoría o no) ¿A qué cree que se debe?

Tratar de recoger información sobre percepción de utilidad de las consultas y modo en cómo lo hacen

\section{T. MI EMBROS DEL EQUI PO: EVOLUCIÓN DE LA ATENCIÓN}

Finalmente, se escribirán las observaciones de los miembros del equipo respecto al enfoque del paciente. Se entiende que no se trata de comentarios de tipo clínico (que se recogerán en el epígrafe correspondiente de la historia clínica) sino respecto a la evolución de las actitudes en la consulta, sugerencias de investigación, precauciones, etc.

a) Evolución de la consulta

b) Precauciones

c) Sugerencias de investigación

d) Otras cuestiones 